EPJ Web of Conferences 41, 03014 (2013)

DOI: $10.1051 /$ epjconf/20134103014

(C) Owned by the authors, published by EDP Sciences, 2013

\title{
Time and momentum resolved resonant magnetic x-ray diffraction on EuTe
}

\author{
C. Trabant ${ }^{1,2}$, N. Pontius ${ }^{1}$, E. Schierle ${ }^{1}$, E. Weschke ${ }^{1}$, T. Kachel ${ }^{1}$, G. Springholz ${ }^{3}$, K. Holldack ${ }^{1}$, \\ A. Föhlisch ${ }^{1}$ and C. Schüßler-Langeheine ${ }^{1}$ \\ ${ }^{1}$ Helmholtz-Zentrum Berlin für Materialien und Energie GmbH, Albert-Einstein Str. 15, Berlin, Germany \\ ${ }^{2}$ II. Physikalisches Institut, Universität zu Köln, Zülpicher Str. 77, Köln, Germany \\ ${ }^{3}$ Institut für Halbleiterphysik, Johannes Kepler Universität, Linz, Austria
}

\begin{abstract}
We used fs- and ps- resonant magnetic x-ray diffraction to probe the laserinduced changes to the magnetic profile in thin films of the antiferromagnetic semiconductor EuTe.
\end{abstract}

\section{Introduction}

Laser induced manipulation of magnetic order on an ultrafast time scale has been a central topic of research for almost the last two decades. The main questions that have to be addressed are that of (a) the relevant processes that are involved in the transfer of energy and angular momentum among the spin, electronic and lattice degrees of freedom in a non-equilibrium state, (b) under which conditions and how fast these processes take place, and (c) how they depend on the material properties [1-3]. Moreover, in view of potential applications the issue of how magnetization dynamics is affected when occurring in confined structures, e.g. in a thin magnetic layer, is of high relevance.

Both of fundamental and of technological interest in this respect are antiferromagnetic materials. They have been discussed to differ from ferromagnets in their ultrafast magnetic properties since no macroscopic magnetization exists and dynamics should not be slowed down by the transfer of angular momentum [4]. In order to explore such effects we studied the prototypical Heisenberg antiferromagnet (AFM) EuTe, which is a semiconductor with a $2.2 \mathrm{eV}$ band-gap. EuTe can be grown as high-quality thin films such that finite-size effects and the influence of interfaces can be probed.

\section{Experiment}

A powerful tool to address antiferromagnetic dynamics is time-resolved resonant magnetic x-ray diffraction (RMXD). Antiferromagnetic order leads to the appearance of superstructure reflections in reciprocal space which are detectable particularly well with soft X-rays tuned resonantly to an excitation into electronic states that carry the magnetic moment. Besides the AFM order parameter also the magnetic depth profile can be determined in this way [5]. The tr-RMXD measurements were performed with a two circle diffractometer equipped with a fast APD-detector at the FEMTOSPEX facility at BESSY II [2]. We studied 40 monolayers (ML) thin films of EuTe sandwiched between either $\mathrm{PbTe}$ or $\mathrm{BaF}_{2}[6]$.

\section{Results and Discussion}

We investigated the photoinduced magnetic dynamics on the fs- and ps-time scale by recording the intensity, peak position and peak width of the $(1 / 21 / 21 / 2)$ antiferromagnetic reflection Fig. 1 shows the loss of AFM order in EuTe represented by the $(1 / 2 / 21 / 2)$ intensity as a function of pump probe delay. The filled Gaussian indicates the temporal resolution of our experiment of about $200 \mathrm{fs}$. Fitting the data with a double exponential function (black line) yields an ultra short time constant of about 700 fs and a second larger one of roughly 5 ps [7].

This is an Open Access article distributed under the terms of the Creative Commons Attribution License 2.0, which permits unrestricted use, distribution, and reproduction in any medium, provided the original work is properly cited. 


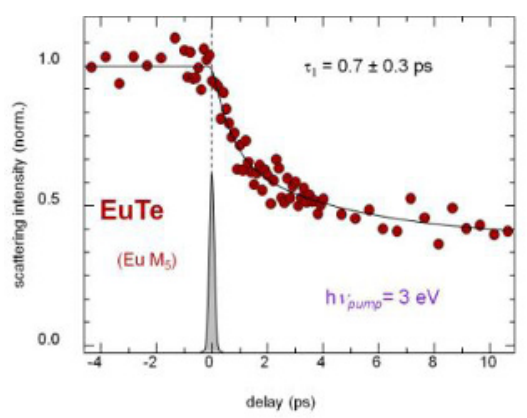

Fig. 1. Decay of the resonant diffraction signal representing the $\mathrm{AF}$ order from the $\mathrm{BaF}_{2}$ - capped sample as a function of pump-probe delay (pump fluence: $\sim 20 \mathrm{~mJ} / \mathrm{cm}^{2}$ ). The black line is a double exponential fit. The filled Gaussian indicates the time resolution in the femtosecond slicing mode.

Momentum resolved scans across the $\left(1 / 2 \frac{1}{2} 2^{1 / 2}\right)$ reflection allow studying the temporal evolution of the spatial magnetic profile across our thin films [5]. In Fig. 2(a-c) the intensity, position and width of the AFM-order central peak in momentum space are plotted as a function of delay measured with fsresolution from 40ML EuTe sandwiched between PbTe. While the peak width, which is inversely proportional to the magnetic correlation length, immediately changes with decreasing peak intensity, the peak position, probing the periodicity, reacts delayed by roughly 10 ps. For larger delays than several tens of ps, the intensity, position and width are stabilized on a transient level. The correlation length is shorter and the periodicity is larger than in thermal equilibrium. Fig. 2(d) shows reciprocal space scans (Laue Profiles) across the magnetic reflection from EuTe, sandwiched between $\mathrm{BaF}_{2}$ for pump probe delays up to $8 \mu \mathrm{s}$. In the top curve the unpumped signal is plotted for comparison. At about $800 \mathrm{~ns}$ after the pump pulse the Laue profiles change significantly. The side maxima have almost vanished and the shift of the main maximum towards smaller q-values is most pronounced. This shift as well as the asymmetric change of the profile has not been observed in equilibrium at any temperature. For delays beyond $8 \mu$ s the q-scans resemble the Laue profile of a EuTe film in thermal equilibrium.
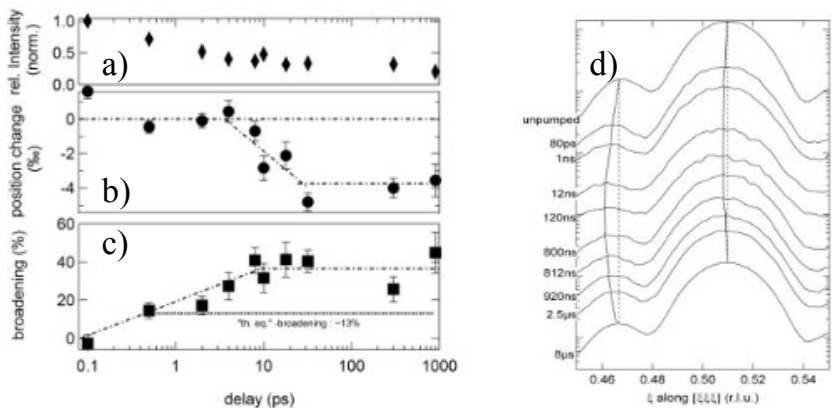

Fig. 2. Fs- and ps- time evolution of the AFM superstructure reflection in momentum space upon laser photo-excitation: (a-c) measured in the PbTe case (pump fluence: $\sim 15 \mathrm{~mJ} / \mathrm{cm}^{2}, \mathrm{E}_{\text {laser }}=1.5 \mathrm{eV}$ ) and (d) in the $\mathrm{BaF}_{2}$ case (pump fluence: $\sim 50 \mathrm{~mJ} / \mathrm{cm}^{2}, \mathrm{E}_{\text {laser }}=3 \mathrm{eV}$ ) (a): relative intensity of the Bragg reflection. (b). Relative change of the main maximum position in momentum space relative to the unpumped case. (c). Relative change of the main peak width compared to the unpumped case. (d) Long term evolution of q-resolved Bragg profiles of the AFM $(1 / 21 / 21 / 2)$ reflection for different pump-probe delays. The dotted lines are guides to the eyes.

\section{Conclusions}

We demonstrate that magnetic order in an AFM-ordered system can be studied by time-resolved RSXD with a time resolution down to $200 \mathrm{fs}$. We found that in EuTe AF order is quenched on timescales of $700 \mathrm{fs}$. Moreover a dramatic change of the Laue profile is found that does not occur at any temperature in thermal equilibrium [5]. The high quality of the momentum-resolved data provide the possibility for real space reconstruction of the magnetic layer profile as a function of pump-probe delay. 


\section{References}

[1] B. Koopmans, G. Malinowski, F. Dalla Longa, D. Steiauf, M. Fähnle, T. Roth, M. Cinchetti, M. Aeschlimann, Nature 9, 259 (2010)

[2] C. Stamm, T. Kachel, N. Pontius, R. Mitzner, T. Quast, K. Holldack, S. Khan, C. Lupulescu, E.F. Aziz, M. Wietstruk, H.A. Dürr, W. Eberhard, Nat. Mat. 6, 740 (2007)

[3] M. Wietstruk, A. Melnikov, C. Stamm, T. Kachel, N. Pontius, M. Sultan, C. Gahl, M. Weinelt, H.A. Dürr, U. Bovensiepen, Phys. Rev. Lett, 106, 127401 (2011)

[4] A. V. Kimel, A. Kirilyuk, A. Tsvetkov, R. V. Pisarev, T. Rasing, Nature 429, 850 (2004)

[5] E. Schierle, E. Weschke, A. Gottberg, W. Sollinger, W. Heiss, G. Springholz, G. Kaindl, Phys. Rev. Lett, 101, 267202 (2008)

[6] G. Springholz, G. Bauer, Phys. Rev. B 48, 10998 (1993)

[7] K. Holldack, N. Pontius, E. Schierle, T. Kachel, V. Soltwisch, R. Mitzner, T. Quast, G. Springholz, E. Weschke, App. Phys. Lett, 97 , 062502 (2010) 